\title{
A comparison of clinical performance between disposable and Goldmann tonometers
}

Royal Eye Hospital, Oxford Road, Manchester M13 9WH, UK

Correspondence: AP Maino, Royal Eye Hospital, Oxford Road, Manchester M13 9WH, UK

Tel: + 44161276 5522;

Fax +441612726618.

E-mail: a.maino@

doctors.net.uk

Received: 9 November 2004 Accepted in revised form:

4 April 2005

Published online:

27 May 2005

This paper was presented orally in an abridged form at the European Glaucoma Society Congress, Florence, 01 June 2004.

Competing interests: None. The Authors have no financial or other interests in any of the products mentioned.

\begin{abstract}
Purpose Applanation tonometry as performed in routine clinical practice is a significant potential vehicle for cross-infection particularly in an emergency eye care setting. The aim of this study is to evaluate the accuracy and reliability of three single-use devices (Tonoshield, Tonosafe, Tonojet) as an alternative to standard Goldmann prisms in an emergency eye department.
\end{abstract}

Methods All patients attending the eye casualty at the Manchester Royal Eye Hospital for a period of 4 months who required intraocular pressure measurement were eligible for this prospective study. Exclusion criteria were: age below 18 years, corneal anomalies that might affect measurement, and refusal to participate. After taking informed consent, the patient was examined by one experienced nurse practitioner, who measured the intraocular pressure three times. In the first part of the study, we compared the standard Goldmann prism $v s$ Tonoshield and Tonosafe prisms, while for the second part of the study we used standard Goldmann, Tonosafe, and Tonojet prisms. Agreement and repeatability tests were carried out on separate samples.

Results Tonosafe and Tonojet correlated well with standard Goldmann tonometry $(P<0.001)$, while the measurements obtained with Tonoshield were higher, especially for raised intraocular pressure measurements. Tonojet and Tonosafe measurements were more reproducible than Tonoshield measurements.

Conclusions This study shows that Tonosafe and Tonojet are accurate and reliable alternatives to standard Goldmann tonometry. Eye (2006) 20, 574-578. doi:10.1038/sj.eye.6701929; published online 27 May 2005
AP Maino, HJ Uddin and AB Tullo

Keywords: disposable prisms; intraocular pressure; cross-infection

\section{Introduction}

The ophthalmologist's consultation room has a significant potential for the transmission of infection. Ophthalmic devices and instruments could be contaminated with bacteria, viruses, or transmissible prions. The role of contaminated ophthalmic equipment is recognised in outbreaks of adenoviral infection, ${ }^{1}$ and it has been demonstrated that hepatitis $B$ infection can be transmitted after infectious exposure of the ocular surface alone. ${ }^{2}$ A number of pathogens such as HIV, Acanthamoeba, hepatitis C virus, herpes simplex virus, have been isolated in human tears ${ }^{3-6}$ and in theory could be spread via ophthalmic equipment.

Although abnormal prion protein has been demonstrated in animal but not the human cornea, it is believed to be transmissible by human corneal transplantation and ophthalmic devices. ${ }^{78}$ Other major problems associated with the current chemical disinfection methods are the extent of damage to the prism after prolonged soaking sessions ${ }^{9}$ or the problem of iatrogenic corneal de-epithelialization. ${ }^{10}$

The only certain way to avoid nosocomial infection via tonometer heads would be adopting single-use devices, as recommended by the Medical Devices Agency. ${ }^{11}$ Silicone tonometer shields (Tonoshield) or disposable tonometer heads (Tonosafe, Tonojet) (Figure 1) therefore might represent an alternative to repeated chemical disinfection of the prism. ${ }^{12,13}$

In the first analysis, we compared Goldmann vs Tonosafe and Tonoshield, as those were the single-use devices most commonly used. The method analysis was repeated when Tonojet become available in the UK. To our knowledge, 

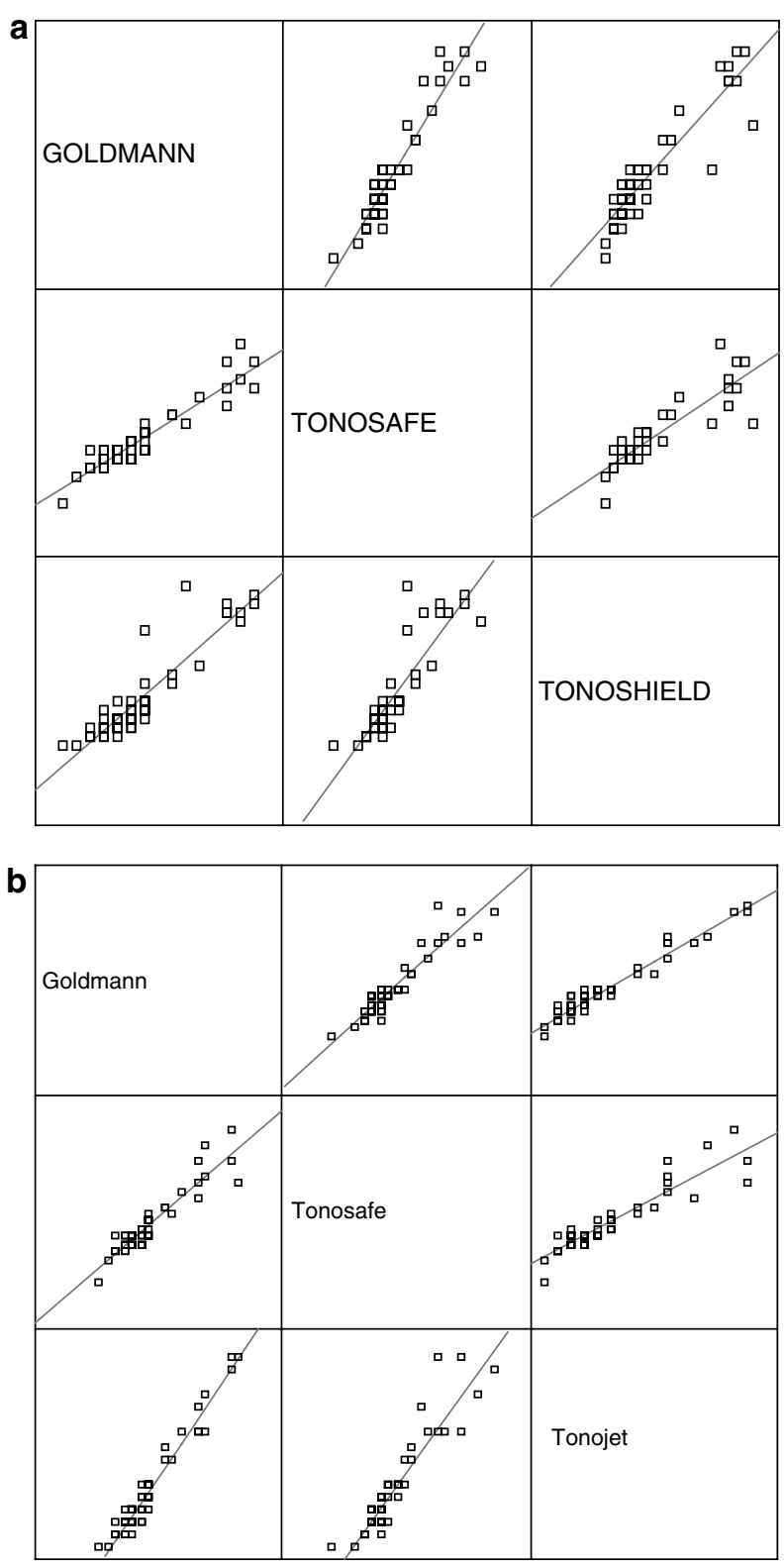

Figure 1 (a) Goldmann vs Tonosafe and Tonoshield. (b) Goldmann vs Tonosafe and Tonojet.

this is the first prospective study to compare different disposable devices vs standard Goldmann tonometry in the setting of an emergency eye clinic.

\section{Materials and methods}

\section{Patient selection}

All patients attending the Emergency Eye Centre at the Manchester Royal Eye Hospital for a period of 4 months who required intraocular pressure (IOP) measurement.
Exclusion criteria were: age below 18 years, presence of corneal abnormalities that might affect IOP measurements (eg epithelial/stromal oedema, large central scars) or might represent a risk factor for corneal abrasions following applanation tonometry (eg corneal dystrophies), active ocular infective disease, blepharospasm, poor subject cooperation, participation to other trials, hospital inpatient status, and refusal to participate. A total of 260 patients met the abovementioned criteria and entered the study. A total of 153 patients (299 eyes) entered two cross-design studies, while 107 patients (187 eyes) were included in ancillary studies (test-retest reliability, agreement).

\section{Methods comparison study No. 1}

After obtaining informed consent, the patient was examined by one experienced nurse practitioner (NP) (HJU), who measured the IOP three times, using the standard Goldmann prism, a Tonosafe prism (Tonosafe, Clement Clarke, UK), and a Tonoshield (Oasis Medical, Glendora, CA, USA). A detailed description of those prisms is available elsewhere. ${ }^{12,13}$

The IOP was measured on the slit-lamp microscope after instillation of a drop of proxymetacaine hydrochloride $0.5 \%$ and fluorescein sodium $0.25 \%$ preservative free solution. In order to avoid errors and bias, the sequence of prisms was determined following a computer-generated random list and the measurements were taken at 5-min intervals. The random order was introduced to eliminate the measurement error that could be introduced with the first measurement.

In order to minimise bias, the slit-lamp breath shield was masked and after each measurement the tonometer dial was adjusted to zero prior to taking another measurement.

\section{Methods comparison study No. 2}

After obtaining informed consent, the investigator (HJU) measured the IOP three times, using the standard Goldmann prism, a Tonosafe prism, and a Tonojet (Tonojet, Luneau, France). The Tonojet device is a singlepiece acrylic applanating prism similar to the standard Goldmann. The manufacturer declares the optical doubling effect to be within International Standards and the device to be latex-free. The prism is available in sterile individual pouches, which allow the mounting of the device on the vertical arm of the Goldmann apparatus without any direct contact and subsequent contamination. The applanating surface of the prism has the same diameter as that of the standard Goldmann. The manufacturer recommends that the slit-lamp 
illumination should be set between 10 and $15^{\circ}$ away from the coaxial position.

\section{Ancillary tests (agreement and precision tests)}

We compared the agreement between the two operators testing a separate sample of 27 patients ( 27 eyes). All the IOP measurements were obtained using the standard Goldmann technique. A test-retest study was carried out for each prism on separate samples of 20 patients (80 patients, 160 eyes in total). For the test-retest study, the same observer (APM) measured the IOP twice using one prism only. All the precautions mentioned before were adopted also in the ancillary studies.

\section{Statistical analysis}

\section{Method comparison}

The tonometry devices (the methods) were compared using Bland-Altman analysis for assessing agreement: ${ }^{14}$ firstly, a scatter-plot matrix was used to compare each pair of methods with the line of perfect agreement; then we plotted the difference against the mean of the two methods. We tested for multiplicative errors using the Bradley-Blackwood test. This test identifies measurement bias that depend on IOP.

\section{Agreement}

A comparison between the two operators was carried out using Bland-Altman plots. Moreover, we also calculated the intraclass correlation as this is an alternative way of expressing agreement between operators.

\section{Precision (repeatability)}

This test is aimed to quantify the repeatability of a method (ie performing applanation tonometry) from replicated measurements obtained with the same prism. This is also known as the test-retest model. We calculated the intraclass correlation coefficient for each method.

\section{Results}

Overall, 260 patients (486 eyes) entered the study. In the first method of comparison analysis (81 patients, 159 eyes), the average measurements were $13.5 \pm 4.03 \mathrm{mmHg}$ for Goldmann, $13.3 \pm 4.11 \mathrm{mmHg}$ for Tonosafe, and $15.1 \pm 4.66 \mathrm{mmHg}$ for Tonoshield. Tonosafe readings correlate well with Goldmann $(P<0.001)$, and there was no evidence for scatter increasing with pressure (regression of difference $v s$ average values, $P=0.47$ ), as shown in Figure 1a. On the other hand, Tonoshield readings were higher $(P<0.001)$, especially for higher IOP $(P<0.001)$.

In the second method (72 patients, 140 eyes), the average measurements were $13.5 \pm 3.5 \mathrm{mmHg}$ for Goldmann, and $13.1 \pm 3.5 \mathrm{mmHg}$ for Tonosafe, and $13.1 \pm 3.5 \mathrm{mmHg}$ for Tonojet (Figure 1b). Both single-use devices correlated well with Goldmann $(P<0.001)$ and no evidence of multiplicative error was found (Bradley-Blackwood test, $P=0.03$ in both cases).

Agreement tests showed that the differences between the two investigators were not significant $(P=0.87)$. An intraclass correlation (ICC) value of 0.97 indicates that $97 \%$ of the variance is attributable to real subject differences and not to observational bias.

Repeatability tests show that Goldmann and Tonosafe are quite reproducible but the Tonoshield is rather less so (ICC Goldmann $=0.989$, ICC Tonosafe $=0.988$, ICC Tonoshield $=0.908$ ). On the other hand, there is no significant difference between Goldmann, Tonosafe, and Tonojet (ICC Goldmann $=0.989$, ICC Tonosafe $=0.984$, ICC Tonojet $=0.989$ ).

\section{Discussion}

The case for the wider use of disposable tonometers is already strong and has been emphasised recently by the demonstration of persistent epithelial cells and protein after the routine cleaning of reusable tonometer heads. ${ }^{15,16}$ However, relatively few studies have been conducted assessing the reliability of disposable tonometers, none yet featuring the Tonojet.

Desai $e t a l^{13}$ found that the recording of IOP by the disposable tonometer prism (Tonosafe) is comparable with that by the standard Goldmann prism. More recently, Salvi et $a l^{17}$ have presented similar findings. On the other hand, Maldonado et $a l^{12}$ reported that the Tonoshield over-read the true intraocular pressure by about $1.9 \mathrm{mmHg}$ whereas Bhatnagar and Gupta ${ }^{18}$ estimated that the silicone shield has a positive bias of $2.09 \mathrm{mmHg}$. A recently published study showed how manufacturing defects can affect Tonosafe prisms. ${ }^{19}$ The authors excluded defective prisms from the analysis and obtained a better agreement between Goldmann and Tonosafe.

While our results were in line with those studies, we found some limitations in the study design, as these studies tested either Tonosafe ${ }^{17}$ or Tonoshield ${ }^{12}$ against the standard Goldmann applanation tonometry and they did not compare one prism $v s$ the other. For this reason, we conducted a method analysis that compared three methods instead of two. This model allowed more refined statistical tests, which lead to a direct comparison between two single-use prisms at the same time. Moreover, none of the previous studies commented 
about the reproducibility of the methods in exam. This means that an important aspect of comparability was overlooked. Comparing two methods that are not precise is difficult as it would not be possible to make assumptions about the amount of agreement. Hence we included ancillary test-retest studies together with method comparison analysis.

Moreover, some studies show obvious additive systematic bias. For instance, Bhatnagar and Gupta ${ }^{18}$ have reported a 'positive' bias of $2.09 \mathrm{mmHg}$. It could be argued that this might depend on systematic errors not identified during the course of the study. Moreover, the Bland-Altman method does not distinguish adequately between fixed and proportional bias and therefore caution should be exercised when interpreting the results. ${ }^{20}$

Another difference between previous studies and ours is in the selection of patients. The studies conducted so far are based on patients who attended general ophthalmic clinics and, where not specified, we can assume that all data were collected by many practitioners. But our study was undertaken in a situation, arguably, more appropriate when the risk of cross-infection is higher, as in emergency departments. For this reason, we selected our sample among casualty attenders only. It could be argued that differences exist between the population attending ophthalmic emergency departments and outpatients services (age range, corneal thickness, ${ }^{21}$ anxiety in first time attenders ${ }^{22}$ ) and that those differences could influence tonometric readings. Another element that we introduced in our study was a single observer in this case a NP. In the UK, NPs are becoming increasingly involved with anterior segment examination and they could be the professional group most affected by a change in practice.

A limitation of our study is that the great majority of our measurements fell in the normal IOP range. Hence, we cannot assume that Tonosafe and Tonojet are a valid alternative to Goldmann for higher IOP readings.

Moreover, the study required repeated IOP measurements and it is well known that this can represent a bias. Whitacre and Stein reported a significant drop in IOP after the first application of a Goldmann tonometer together with a consensual response in the contra-lateral eye. However, they concluded that alternating measurements between the two eyes (after discarding the first reading) is an accurate method..$^{22}$ For this reason the pressure measurements were be taken seven times, with the first reading excluded from the analysis. In conclusion, Tonosafe and Tonojet represent reliable alternatives to Goldmann tonometers in the setting of an emergency eye department. Further studies are needed to demonstrate the role of single use devices in specialist Glaucoma clinics.

\section{Acknowledgements}

We thank Dr S Roberts, Department of Biostatistics, University of Manchester, UK for his expert statistical advice.

\section{References}

1 Gordon YJ, Gordon RY, Romanowski E, Araullo-Cruz TP. Prolonged recovery of desiccated adenoviral serotypes 5, 8 and 19 from plastic and metal surfaces in vitro. Ophthalmology 1993; 100: 1835-1839.

2 Su CS, Bowden S, Fong LP, Taylor HR. Current tonometer disinfection may be inadequate for hepatitis B virus. Arch Ophthalmol 1994; 112: 1406-1407.

3 Segal WA, Pirnazar JR, Arens M, Pepose JS. Disinfection of Goldmann tonometers after contamination with hepatitis C virus. Am J Ophthalmol 2001; 131: 184-187.

4 Pepose JS, Linette G, Lee SF, MacRae S. Disinfection of Goldmann tonometers against human immunodeficiency virus type 1. Arch Ophthalmol 1989; 107: 983-985.

5 Smith CA, Pepose JS. Disinfection of tonometers and contact lenses in the office setting: are current techniques adequate? Am J Ophthalmol 1999; 127: 77-84.

6 Yamamoto S, Shimomura Y, Kinoshita S, Nishida K, Yamamoto R, Tano Y. Detection of herpes simplex virus DNA in human tear film by the polymerase chain recation. Am J Ophthalmol 1993; 117: 160-163.

7 Walia JS, Chronister CL. Possible iatrogenic transmission of Creutzfeldt-Jakob disease via tonometer tips: a review of the literature. Optometry 2001; 72: 649-652.

8 Macalister GO, Buckley RJ. The risk of transmission of Creutzfeldt-Jakob disease via contact lenses and ophthalmic devices Contact Lens Ant Eye 2002; 25: 104-136.

9 Key C, Whitman J. Alcohol soaking damages applanation tonometer heads. Arch Ophthalmol 1986; 104: 800.

10 Levenson J. Corneal damage from improperly cleaned tonometer tips. Arch Ophthalmol 1989; 107: 1117.

11 MDA AN1999 (03): Single patient use of ophthalmic medical devices: implications for clinical practice. Medical Devices Agency, October 1999.

12 Maldonado MJ, Rodriguez-Galietero A, Cano-Parra J, Menezo JL, Diaz-Llopis M. Goldmann applanation tonometry using sterile disposable silicone tonometer shields. Ophthalmology 1996; 103: 815-821.

13 Desai SP, Sivakumar S, Fryers PT. Evaluation of a disposable prism for applanation tonometry. Eye 2001; 15: 279-282.

14 Bland JM, Altman DG. Measuring agreement in method comparison studies. Stat methods Med Res 1999; 8: 135-160.

15 Lim R, Dhillon B, Kurian KM, Aspinall PA, Fernie K, Ironside JW. Retention of corneal epithelial cells following Goldmann tonometry: implications for CJD. Br J Ophthalmol 2003; 87: 583-586.

16 Amin SZ, Smith L, Luthert PJ, Cheetham ME, Buckley RJ. Minimising the risk of prion transmission by contact tonometry. Br J Ophthalmol 2003; 87: 1360-1362.

17 Salvi SM, Sivakumar S, Sidiki SS. Use of disposable prism tonometry in routine clinical practice. Eye, Advance online publication 10 September 2004. 
18 Bhatnagar A, Gupta AK. Disposable devices for measuring intraocular pressure: a clinical study to assess their accuracy. Eye, Advance online publication 10 September 2004.

19 Goel S, Chua C, Dong B, Butcher M, Ahfat A, Hindi SK et al. Comparison between standard Goldmann applanation prism and disposable applanation prism in tonometry. Eye 2004; 18: 175-178.
20 Ludbrook J. Statistical techniques for comparing measures and methods of measurement: a critical review. Clin Exp Pharm Physiol 2002; 29: 527-536.

21 Brandt JD, Beiser JA, Kass MA, Gordon MO. Central corneal thickness in the Ocular Hypertension Treatment Study (OHTS). Ophthalmology 2001; 108: 1779-1788.

22 Whitacre MM, Stein R. Sources of error with use of Goldmann-type tonometers. Surv Ophthalmol 1993; 38: 1-30. 